Z. Yin, B. Font, G. Eckel, C. Abram, W. Meier, I. Boxx, and F. Beyrau, Investigation of $\mathrm{BAM}: \mathrm{Eu}^{2+}$ particles as a tracer for temperature imaging in flames, Combustion and Flame 184 (2017) 249-251

The original publication is available at www.elsevier.com

https://doi.org/10.1016/j.combustflame.2017.06.005 


\title{
Investigation of $\mathrm{BAM}: \mathrm{Eu}^{2+}$ particles as a tracer for temperature imaging in flames
}

\author{
Zhiyao Yin ${ }^{\mathrm{a}, 1}$, Benoit Fond ${ }^{\mathrm{b}, 1, *}$, Georg Eckel ${ }^{\mathrm{a}}$, Christopher Abram ${ }^{\mathrm{b}}$, Wolfgang Meier ${ }^{\mathrm{a}}$, Isaac Boxx ${ }^{\mathrm{a}}$, \\ Frank Beyrau ${ }^{\mathrm{b}}$ \\ ${ }^{a}$ Institute of Combustion Technology, German Aerospace Center (DLR), Stuttgart, Germany \\ ${ }^{b}$ Lehrstuhl für Technische Thermodynamik, Otto-von-Guericke-Universität Magdeburg, Magdeburg, Germany
}

\begin{abstract}
The capabilities of the joint temperature-velocity imaging technique, thermographic particle image velocimetry (TPIV) are examined in $\mathrm{H}_{2}$ laminar diffusion flames, by seeding the fuel stream with $\mathrm{BAM}: \mathrm{Eu}^{2+}$ particles. The upper measurable temperature is found to be 900-1000 K. For these phosphor particles this limit is primarily imposed by thermal quenching of the laser-induced luminescence emission that is used for thermometry. Within this limit, the measured temperature distribution quantitatively agrees with a numerical simulation. It is also shown that the luminescence signal per phosphor particle is not influenced by passing the particles through a high temperature reaction zone.
\end{abstract}

\section{Introduction}

Accurate, spatially-resolved temperature measurements are much sought after in combustion research. The task remains a challenge in many environments of interest despite advancements in laser-based thermometries. The recently-emerged thermographic particle image velocimetry (TPIV) presents a viable alternative [1 6]. It uses micrometer-sized thermographic phosphor particles seeded into the flow as a tracer. A typical setup involves a two-colour emission intensity ratio approach, whereby temperature-dependent laser-induced luminescence from the seeded particles is spectrally filtered to determine the particle temperature, which equilibrates rapidly with carrier gas temperature [1. Unlike organic tracers, thermographic phosphor particles are inert and have high melting points, and the luminescence properties are generally insensitive to gas composition [7, 8], making the temperature measurement independent of the local environment. Additionally, using a standard PIV approach, the velocity field can be simultaneously measured using laser Mie scattering from the same particles. This lends TPIV the potential to study heat transfer and the interaction between turbulence and chemistry in combustion environments.

However, applications of TPIV in practical flame environments may be limited by (1) the relatively narrow range of measurable temperature and (2) the thermal degradation of the phosphor particles. The latter is critical as the seeding is sometimes achieved via flow recirculation in enclosed flames where particles may go through high (unmeasurable) temperature regions before they reach low (measurable) temperature regions. In this work, we examined these issues by applying TPIV using a well-documented phosphor BAM : $\mathrm{Eu}^{2+}$ [1, 5, 8, 9] in $\mathrm{H}_{2}$ laminar diffusion flames.

\footnotetext{
${ }^{*}$ Corresponding author

Email address: benoit.fond@ovgu.de (Benoit Fond)

${ }^{1}$ These authors contributed equally to this work.
} 
Previously, it was shown that, for $\mathrm{BAM}: \mathrm{Eu}^{2+}$ particles, the temperature calibration remained unchanged before and after combustion in a vessel [9]. This corresponded to an exposure of $0.5 \mathrm{~s}$ between 1400 and $2000 \mathrm{~K}$. In a study of much longer exposures $(20 \mathrm{~min}$ ) at $1470 \mathrm{~K}$, only a subtle change in the room temperature emission spectrum was observed [10], translating to an $\sim 80 \mathrm{~K}$ calibration error. Therefore, it is reasonable to assume that short exposure to high temperatures does not alter the luminescence emission spectrum.

However, in Refs [10, 11], exposures of several minutes at these temperatures caused the luminescence emission intensity to decrease by orders of magnitude, a phenomenon referred to as thermal degradation. This second phenomenon has not yet been investigated for short exposure times relevant to reactive flows, though it would be problematic since it would compromise the measurement precision. Therefore the objectives of this work are to determine (1) the maximum measurable temperature and (2) the luminescence emission intensity of BAM : $\mathrm{Eu}^{2+}$ particles after passage through a high temperature reaction zone. In addition, a CFD simulation was conducted to provide a quantitative comparison to the measured temperatures.

\section{Experimental setup}

A standard Gülder burner (with ID=10.9 mm inner nozzle and ID=100 mm co-flow [12, 13]) with a fuel mixture of $\mathrm{N}_{2} / \mathrm{H}_{2} / \mathrm{CH}_{4}$ was chosen to generate attached laminar diffusion flames. A small amount of $\mathrm{CH}_{4}$ was introduced to visualise and monitor flame stability. The fuel stream was seeded with BAM : $\mathrm{Eu}^{2+}$ particles (Phosphor Technology KEMK63-UF/P1 with volume-equivalent sphere diameters of $1.2 \mu \mathrm{m}$ for $d_{5}, 2.4 \mu \mathrm{m}$ for $d_{50}$ (median) and $4.1 \mu \mathrm{m}$ for $d_{95}$ by volume) using a magnetically stirred seeder (LaVision Particle Blaster 100). Note that part of the seeded fuel was diverted directly to the exhaust to maintain a small flow rate (1-2.5 SLM) for a short and stable flame. The co-flow was not seeded to ensure that all the particles would go through the reaction zone, and also to avoid interference from multiple scattering [5].

The TPIV setup is similar to those in Refs. [1, 4, 14]. The 355-nm output of a single-cavity double-pulse Nd:YAG laser $(5 \mathrm{~Hz})$ was formed into a light sheet $(50 \mathrm{~mm} \times 0.6 \mathrm{~mm})$ at the center of the fuel nozzle. For temperature imaging, luminescence from the particles following the first pulse only $\left(38 \mathrm{~mJ} / \mathrm{cm}^{2}\right)$ was detected by two hardware-binned $(4 \times 4)$ CCD cameras with $50-\mathrm{mm} \mathrm{f} / 1.4$ lenses, interference filters (466 $\pm 40 \mathrm{~nm}$ and 355-nm notch) and a dichroic beamsplitter (455-nm longpass), as recommended by the analysis of Ref.[8]. On the opposite side, an sCMOS camera was installed for 2-component PIV using Mie scattering from the same phosphor particles at $355 \mathrm{~nm}$. In addition the Mie scattering images were used to determine the local seeding density field in the measurement plane, after calibration of the system at room temperature using the luminescence intensity per particle data from Ref. [8].

For thermometry, a cutoff filter (15 counts) and a $3 \times 3$ moving average filter were applied to the background-subtracted luminescence images to improve signal to noise ratio for a final inplane resolution of $600 \mu \mathrm{m}$. 2D intensity ratio was obtained from each pair of images and was normalised by an average ratio obtained at room temperature to account for flat fielding as well as non-uniformity in the excitation laser sheet [14]. The intensity ratio was converted to temperature using a calibration curve derived from powder-based spectral measurements inside a furnace with optical access [8].

\section{Numerical simulation}

Steady state simulations of the experimental cases were conducted using a DLR in-house code THETA (a 3D finite volume solver optimised for combustion problems [15]) on a fully-structured 
hexahedral mesh with ca. 610000 elements. The mesh was refined in the reaction zone $(>20$ grid points) as well as close to the nozzle exit.

The $\mathrm{H}_{2}$ chemical kinetics mechanism was taken from Ref.[16]. The chemical reactions were calculated by a direct chemistry solver. Gravity and differential diffusion were also accounted for [17]. The measured velocity profile near the burner nozzle exit was used as a boundary condition in the simulation. The small amount of $\mathrm{CH}_{4}$ added in the experiment was neglected in the simulation.

\section{Results}

Figure 1a\&b compares a single-shot measured temperature distribution to a simulated steadystate temperature for a $\mathrm{N}_{2} / \mathrm{H}_{2} / \mathrm{CH}_{4}(66 \% / 33 \% / 1 \%)$ diffusion flame. The flame was stable for these conditions. Since the co-flow was not seeded, only the inner core region can be measured. From the extracted radial temperature profiles in Fig.2, it is clear that the current implementation of TPIV results in an upper measurable temperature of 900-1000 K. Within this limit, the agreement between the measured and simulated temperature appears excellent.

Figure 1 $\&$ d show the luminescence signal from the particles (one channel) and measured seeding density from a single-shot measurement. As temperature initially increases with distance from the nozzle exit, the signal intensity reduces and disappears as the particles penetrate further into the reaction zone, even though there is still a moderate level of seeding $\left(3 \times 10^{11}\right.$ particles $/ \mathrm{m}^{3}$ at $1000 \mathrm{~K}$ ). It is clear that thermal quenching rather than seeding density limits the range of measurable temperature. A phosphor with a more favorable thermal quenching behaviour is needed to increase the upper temperature limit of the technique.

The luminescence intensity of the seeded phosphor particles was measured downstream of the reaction zone in a $\mathrm{H}_{2} / \mathrm{CH}_{4}(96.9 \% / 3.1 \%)$ diffusion flame. The measurement field of view was $20 \mathrm{~cm}$ above the burner nozzle exit. Patches of luminescence signal were captured in this region, corresponding to the emission from particles that have gone through the hot reaction zone and cooled by the ambient. From the numerical simulation, the residence time of the particles in the diffusion flames in regions exceeding $1700 \mathrm{~K}$ (up to a maximum of $2400 \mathrm{~K}$ ) was estimated to be 0.5-1 s. The temperature of these patches was evaluated using the same calibration data as the aforementioned studies showed little effect of high temperature exposure on the emission spectrum. Figure 3 compares the mean luminescence signal per particle from these patches to measurement results in heated jets from Ref. [8]. It is clear that the luminescence signal of the particles past the reaction zone matches that of particles in heated jets, indicating negligible influence from the exposure to high temperatures in the reaction zone. For practical relevance, in an extensivelyresearched atmospheric swirl-stabilised flame with an equivalence ratio of 0.7 and a thermal power of $25 \mathrm{~kW}$ [18, a residence time of $0.1 \mathrm{~s}$ was estimated based on the experimental results from Ref. [19] for particles residing in the central recirculation zone, which has an average temperature of $1700 \mathrm{~K}$.

\section{Conclusions}

To summarise, in $\mathrm{H}_{2}$ laminar diffusion flames, temperatures up to 900-1000 K were accurately measured using thermographic particle image velocimetry (TPIV) with BAM : Eu ${ }^{2+}$ phosphor particles. The luminescence emission intensity of the particles was also found to be unaffected after they went through the reaction zone of a laminar non-premixed flame. The result suggests that TPIV with BAM : $\mathrm{Eu}^{2+}$ particles may be readily applicable to enclosed flames, specifically for simultaneous velocity and temperature measurements in low-medium temperature regions where fuel injection and mixing with hot exhaust gases occurs. 


\section{Acknowledgment}

Zhiyao Yin acknowledges the financial support within the Helmholtz Postdoc Programme (Grant PD-112).

\section{References}

[1] B. Fond, C. Abram, A. L. Heyes, A. M. Kempf, F. Beyrau, Opt. Express 20 (2012) 22118-22133.

[2] A. Omrane, P. Petersson, M. Aldén, M. Linne, Applied Physics B 92 (2008) 99-102.

[3] N. J. Neal, J. Jordan, D. Rothamer, SAE Int. J. Engines 6 (2013) 300-318.

[4] C. Abram, B. Fond, A. Heyes, F. Beyrau, Appl. Phys. B 111 (2013) 155-160.

[5] H. Lee, B. Böhm, A. Sadiki, A. Dreizler, Applied Physics B 122 (2016) 1-13.

[6] L. Fan, Y. Gao, A. Hayakawa, S. Hochgreb, Experiments in Fluids 58 (2017) 34.

[7] J. Brübach, A. Dreizler, J. Janicka, Measurement Science and Technology 18 (2007) 764.

[8] B. Fond, C. Abram, F. Beyrau, Applied Physics B 121 (2015) 495-509.

[9] J. P. J. van Lipzig, M. Yu, N. J. Dam, C. C. M. Luijten, L. P. H. de Goey, Applied Physics B 111 (2013) 469-481.

[10] A. Yáñez-González, B. van Wachem, S. Skinner, F. Beyrau, A. Heyes, Materials and Design 108 (2016) $145-150$.

[11] Y. H. Wang, Z. H. Zhang, Electrochemical and Solid-State Letters 8 (2005) H97-H99.

[12] C. Schulz, B. Kock, M. Hofmann, H. Michelsen, S. Will, B. Bougie, R. Suntz, G. Smallwood, Applied Physics B 83 (2006) 333-354.

[13] R. Hadef, K. P. Geigle, J. Zerbs, R. A. Sawchuk, D. R. Snelling, Applied Physics B 112 (2013) 395-408.

[14] C. Abram, B. Fond, F. Beyrau, Opt. Express 23 (2015) 19453-19468.

[15] T. Blacha, M. D. Domenico, P. Gerlinger, M. Aigner, Combustion and Flame 159 (2012) 181 $-193$.

[16] M. Ó Conaire, H. J. Curran, J. M. Simmie, W. J. Pitz, C. K. Westbrook, International Journal of Chemical Kinetics 36 (2004) 603-622.

[17] M. Di Domenico, Numerical Simulations of Soot Formation in Turbulent Flows, Ph.D. thesis, Fakultät für Luft- und Raumfahrttechnik und Geodäsie, Universität Stuttgart, 2008.

[18] W. Meier, P. Weigand, X. Duan, R. Giezendanner-Thoben, Combustion and Flame 150 (2007) $2-26$.

[19] L. Cheng, A. Spencer, Experiments in Fluids 52 (2012) 647-661. 

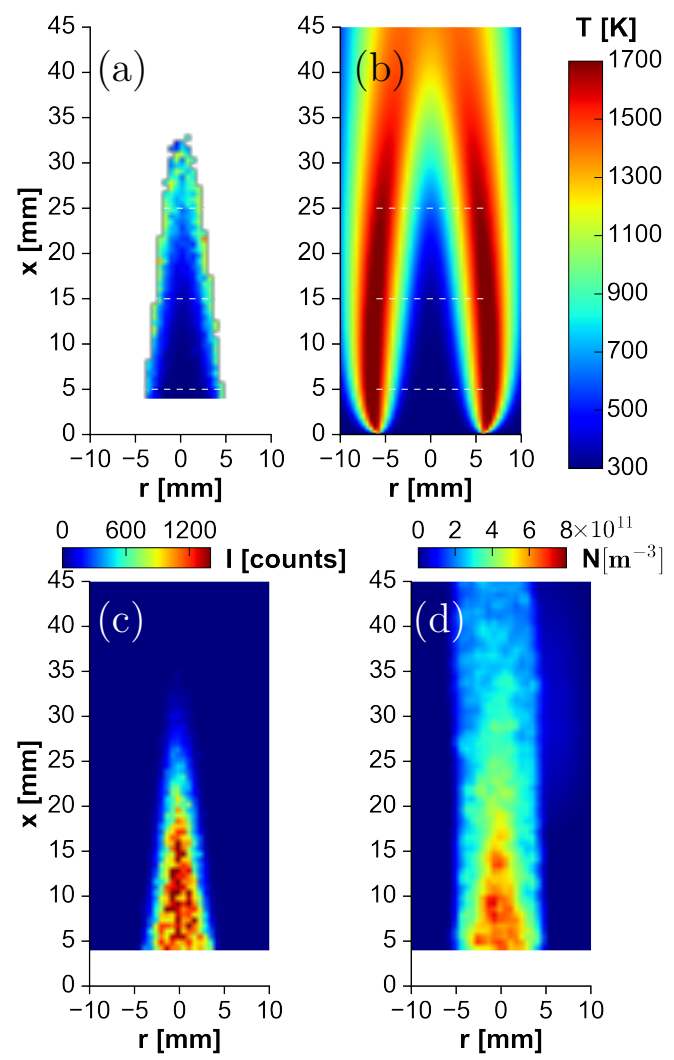

Figure 1: (a, c, d) Single-shot measured temperature field, luminescence signal (one channel), and measured seeding density obtained in an $\mathrm{N}_{2} / \mathrm{H}_{2} / \mathrm{CH}_{4}$ $(66 \% / 33 \% / 1 \%)$ diffusion flame $\left(\mathrm{U}_{\mathrm{avg}}=0.7 \mathrm{~m} / \mathrm{s}\right)(\mathrm{b}) \mathrm{Sim}-$ ulated temperature field for the same flame

Figure 3:

Luminescence signal per particle as measured in heated jets (taken from Ref. [8]) and downstream of a $\mathrm{H}_{2} / \mathrm{CH}_{4}(96.9 \% / 3.1 \%)$ diffusion flame $\left(\mathrm{U}_{\mathrm{avg}}=0.5 \mathrm{~m} / \mathrm{s}\right)$. The error bars represent the statistical uncertainty of the mean intensity per particle (vertical), and mean temperature (horizontal), averaged over 66 recorded patches, i.e $\frac{\sigma}{\sqrt{66}}$ ( $\sigma$ is the patch-topatch standard deviation)

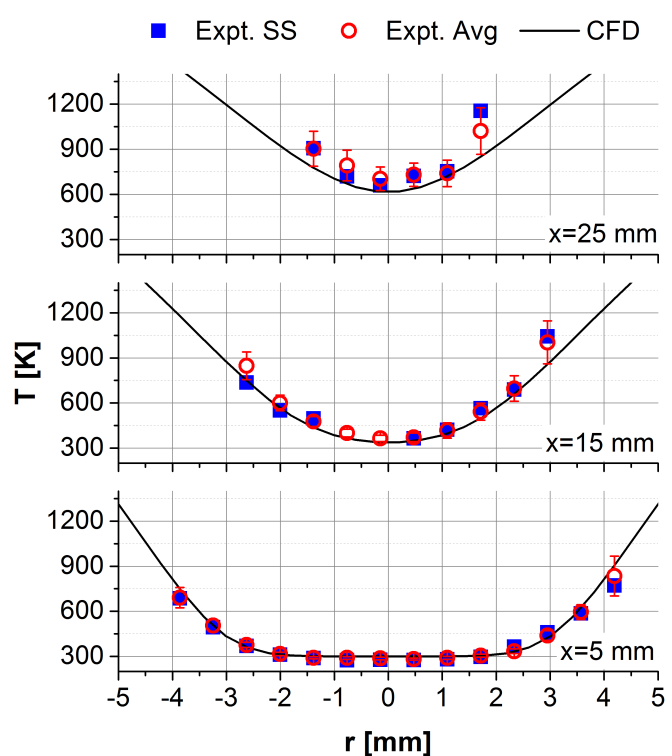

Figure 2: Radial temperature profiles at various axial locations (dashed lines in Fig 1) extracted from the measurement and simulation results shown in Fig.1. Square symbols: one single-shot measurement. Circle symbols with error bars: average and standard deviation of 25 single-shot measurements.

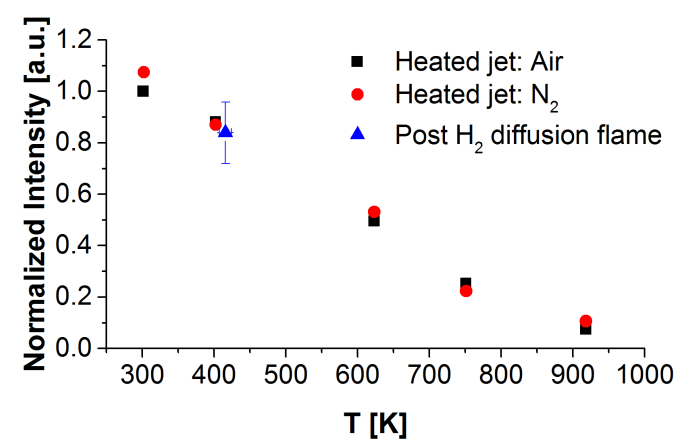

